\title{
Physiological Effect of Laccase, Secreted by Rigidoporus microporus on the Host Pathogen Interactions
}

\author{
H.K.I. Madushani ${ }^{1}$, T.H.P.S. Fernando ${ }^{1}$, R.L.C. Wijesundara ${ }^{2}$, P. Senaviratne ${ }^{1}$ \\ ${ }^{I}$ Department of Plant Pathology and Microbiology, Rubber Research Institute, Dartonfield, \\ Agalawatta, Sri Lanka \\ ${ }^{2}$ Department of Plant Science, University of Colombo, Sri Lanka \\ *thpsfernando@yahoo.com
}

\begin{abstract}
Laccases (EC1.10.3.2) are multi-copper containing phenol oxidases that catalyse the oxidation of phenolic compounds and aromatic amines. They are widely distributed in higher plants and in many fungi. In fungi, they are involved in delignification, sporulation, pigment production and plant pathogenesis and fruiting body formation. Rigidoporus microporus is a white rot fungus efficient lignin degradation and causative agent of the most destructive white root disease. In this study, the production of laccase was detected qualitatively. Furthermore involvement of laccase in the host-pathogen interaction was also studied. Three isolates of $R$. microporus were isolated from symptomatic Hevea brasiliensis (R1), Mucuna bractiata (R2), and Artocarpus nobilis (R3). The level of laccase production was measured qualitatively on solid media containing the colour indicator of guaiacol. $5 \mathrm{~mm}$ diameter of mycelium discs from 7 day old cultures were innoculated into 2\% PDA plates supplemented with $0.2 \%$ glucose as a laccase degrading substrate with $0.02 \%$ Guaiacol $(\mathrm{pH} \mathrm{5.5)}$ and incubated at RT $\left(28 \pm 2^{0} \mathrm{C}\right)$ under continuous dark conditions. After, an incubation period of 4 days, the enzyme activity was examined by the decolorisation intensity. Morphology of the three isolates was also evaluated on malt extract agar. Lignin degradation ability was studied using artificial inocula prepared by fresh root pieces of $\mathrm{H}$. brasiliensis and incubated for four months; thereafter they were splited longitudinally to observe the lignin degradation ability of the isolates. All the test isolates were laccase positive and R3 (A. nobilis) isolate produced higher intensity showing comparatively larger diameter of the reddish brown halos indicting higher level of laccase production. Moreover, the isolate R3 showed the formation of rhizomorphs in vitro conditions while the other isolates did not show them. Formation of rhizormorphs is associated with the laccase synthesis which has been reported to be responsible for making polyphenolic glue that sticks the hyphae together. The degradation of roots was observed to be comparatively faster by the isolate $\mathrm{R} 3$ leaving white pockets on roots by degrading cell wall component due to the removal of lignin in localized areas of wood and observation can be attributed with the higher laccase enzyme. The root pieces inoculated with R1 and R2 did not show white pockets and a spongy nature until four month incubation period. It has been revealed that the isolate R3 (A. nobilis) is a good laccase producer among the other isolates under investigation.
\end{abstract}

Keywords: Laccase, Rhizormorphs, Rigidoporus microporus

Acknowledgement: Authors acknowledge the financial assistance of National Research Council (Grant No. 11-39)

Proceedings of the International Forestry and Environment Symposium 2015 of the Department of Forestry and Environmental Science, University of Sri Jayewardenepura, Sri Lanka 